\title{
Formation of Hydroxyeicosatetraenoic Acids (HETE) in Blood From Adults Versus Neonates: Reduced Production of 12-HETE in Cord Blood
}

\author{
RONALD W. WALENGA, ${ }^{1}$ SHIRAZ SUNDERJI, AND MARIE J. STUART \\ Department of Pediatrics [R.W.W., M.J.S.] and Obstetrics and Gynecology [S.S.], State University of New York \\ Health Science Center, Syracuse, New York 13210
}

\begin{abstract}
Hydoxyeicosatetraenoic acids (HETE) are major arachidonic acid metabolites of a number of cells found in blood and blood vessels. These products have been implicated in physiologic responses as diverse as platelet aggregation, cell migration, and cell proliferation. Using a sensitive and specific assay, GC/selected ion monitoring after high-performance liquid chromatography separation, we have measured the levels of three HETE isomers of biologic significance 12-HETE, 15-HETE, and 5-HETE in plasma, serum and stimulated serum (formed in the presence of arachidonic acid and calcium ionophore), obtained from normal adults and cord blood from normal neonates. Whereas there were no significant differences between the two groups for 5- or 15-HETE in any of the samples, stimulated serum from adults produced 12 times as much 12-HETE when compared to cord blood. When platelets were isolated from adult and cord blood, 12HETE production by neonatal platelets, stimulated with $10 \mu \mathrm{M}$ arachidonic acid, was less than one-fourth that of adults. Although no role for 12-HETE in normal platelet responses has yet been established, it has been reported that those individuals with myeloproliferative syndromes who demonstrate a concomitant decrease in platelet 12HETE synthetic ability have an increased bleeding tendency. It needs to be further evaluated if this already depressed level of 12-lipoxygenase in neonatal platelets may contribute to pathologic bleeding in those infants subjected to additional stress (such as prematurity or birth asphyxia). (Pediatr Res 24: 563-567, 1988)
\end{abstract}

\section{Abbreviations}

HETE, hydroxyeicosatetraenoic acid HPLC, high-performance liquid chromatography PPP, platelet-poor plasma

Hydroxyeicosanoids are synthesized by many of the formed elements of blood. In fact, the major arachidonic acid metabolite produced by platelets is 12-HETE (1). Neutrophils and macrophages produce 5-HETE as a side-product in the biosynthesis of

Received June 9, 1987; accepted June 29, 1988

Correspondence and reprint requests M. J. Stuart, Hematology, St. Christopher's Hospital for Children, 5th and Lehigh Avenue, Philadelphia, PA 19133.

Supported by Public Health Service Grants HD-14405 and EY-05614 to M.J.S. and an American Heart Association, New York Affililiate grant to R.W.W.

${ }^{1}$ Present address Department of Pediatrics, St. Christopher's Hospital for Children, 5th and Lehigh Avenue, Philadelphia, PA 19025. leukotrienes (2) and can synthesize 15-HETE (3). In addition, vascular endothelial cells have been shown to produce 15-HETE $(4,5)$. Although the discovery of these compounds predates the discovery of such important eicosanoids as thromboxane $A_{2}$, prostacyclin, and the leukotrienes, there is no clear-cut biologic function ascribed to them. Work in this laboratory, and in others, has suggested a role for such compounds in modulating migration (6-9) and proliferation (10) of endothelial cells that line the vascular wall. Additionally, there are suggestions that some HETE modulate the production of agents such as thromboxane and prostacyclin, which directly participate in platelet-vessel interactions (11).

In ${ }^{14} \mathrm{C}$-arachidonic acid prelabeled platelets, using strong agonists, we have previously demonstrated differences in arachidonic acid release and metabolism between neonatal and adult platelets (12). No data are available on leukocyte or monocyte arachidonate metabolism in the neonate. We therefore have investigated the levels of the various HETE of biologic significance found in adult and neonatal plasmas and the levels of these compounds in serum formed either spontaneously, or when stimulated with levels of arachidonic acid and calcium ionophore found to maximize HETE production in adult platelets.

\section{MATERIALS AND METHODS}

Donors and collection of blood. Blood was drawn by venipuncture from 12 normal adults (six females, six males) between the ages 23 and 47 . After informed consent of the mother, umbilical venous blood was drawn from 16 normal, full-term neonates (described in Table 1). Immediately after delivery (either vaginally or by cesarean section) clamps were placed on the umbilical cord, a 19-gauge needle was introduced into the umbilical vein near the placenta, and blood was withdrawn by a two-syringe technique. None of the adult donors or mothers had ingested any medications known to effect cyclooxygenase or lipoxygenase activity. White blood cell and platelet counts were determined to be in the normal range.

Aliquots were collected into tubes containing no anticoagulant to form serum, or tubes containing $2 \mathrm{mM}$ arachidonic acid and $25 \mu \mathrm{M}$ A23187 to produce stimulated serum. Preliminary experiments with adult samples had demonstrated that the presence of arachidonic acid during serum formation increased 12-HETE production and that the stimulation was maximal between 1 and $2 \mathrm{mM}$ arachidonate. Further stimulation was produced by the calcium ionophore A23187, and this stimulation was maximal at $25 \mu \mathrm{M}$. These tubes were immediately brought to $37^{\circ} \mathrm{C}$ and maintained at that temperature for $1 \mathrm{~h}$. Sera were then prepared by centrifugation at $1200 \times g$ for $5 \mathrm{~min}$. Additional aliquots were collected in tubes anticoagulated with $0.0894 \mathrm{M}$ bisodium 
Table 1. Vital statistics of cord blood donors

\begin{tabular}{lccrr}
\hline \multicolumn{1}{c}{ Parameter } & Mean & SEM & Maximum & Minimum \\
\hline Gestational age (wk) & 39.4 & 0.3 & 42 & 36 \\
Birth wt (g) & 3648 & 131 & 4820 & 2837 \\
Apgar 1 & 8.1 & 0.2 & 9 & 6 \\
Apgar 2 & 8.9 & 0.2 & 10 & 7 \\
Platelets $\left(10^{3} / \mu 1\right)$ & 294 & 19 & & \\
White cells $\left(10^{3} / \mu \mathrm{l}\right)$ & 12.7 & 1.4 & & \\
Delivery & & & & \\
$\quad$ Vaginal & 3 & & & \\
$\quad$ Cesarean & 13 & & & \\
Sex & & & & \\
$\quad$ Male & 8 & & & \\
$\quad$ Female & 8 & & & \\
\hline
\end{tabular}

citrate, $0.0156 \mathrm{M}$ citric acid, $0.016 \mathrm{M}$ citric acid and $0.01418 \mathrm{M}$ dextrose. PPP was then prepared by centrifugation at $1200 \times g$ for $10 \mathrm{~min}$ in a Beckman J6B centrifuge (Beckman Instruments, Fullerton, CA). A plasma sample was also incubated with arachidonate and A23187 to control for noncell-mediated production of HETE under these conditions.

Extraction and isolation of HETE from blood samples. Each of the samples was treated with an equal volume of ice cold $\left(-4^{\circ}\right.$ C) ethanol (to precipitate protein), acidified with one-tenth volume of $1.0 \mathrm{~N} \mathrm{HCl}$ and extracted with five volumes of ethyl acetate. The ethyl acetate phase was removed and replaced with an equal volume of ethyl acetate and the extraction repeated. The combined ethyl acetate phases were dried over anhydrous $\mathrm{MgSO}_{4}$, and evaporated under a nitrogen stream. The extracts were dissolved in a minimal volume of $\mathrm{CHCl}_{4}$ and applied to a dry-packed silicic acid column $(1 \mathrm{~g} /$ each $5 \mathrm{ml}$ or smaller portion of serum). The columns were washed with 10 column volumes of hexane/ether (9:1), to elute di- and triglycerides, nonhdyroxylated fatty acids, and other neutral lipids. The column was then eluted with 10 column volumes of hexane/ether (50:50). This fraction contained the monohydroxy-eicosanoids. Solvent was removed without heating under reduced pressure in a Savant Speed Vac sample concentrator.

Before HPLC analysis, the sample was dissolved in methanol, diluted with water to a final concentration of $75 \%$ methanol, then injected onto a Waters $\mathrm{C}_{18}$-reverse phase column (Waters Associates, Milford, MA). The column was eluted with $72 \%$ methanol $/ 28 \%$ acetic acid $(0.1 \%)$ at a flow of $1.0 \mathrm{ml} / \mathrm{min}$, a modification of the method of Borgeat et al. (13). Column effluent was monitored at $\mathrm{A}_{237} \mathrm{~nm}$. Fractions corresponding to the retention regions of authentic standards of 5-HETE, 12HETE, and 15-HETE were collected for GC/MS analysis. An appropriate amount of ricinoleic acid (usually $1.0 \mathrm{nmol}$ ) was added as an internal standard to each peak before derivatization.

Derivatization of samples and standards for GC/SIM. Standard curves were generated from ricinoleic acid $(1.0 \mathrm{nmol})(\mathrm{Ap}-$ plied Sciences, Belefonte, PA) mixed with known amounts (from 0.01 to $3.0 \mathrm{nmol}$ ) of either 5-, 12-, or 15-HETE. Standards were purchased from either Biomol (Philadelphia, PA) or Cayman (Ann Arbor, MI). Standard purity was assessed by reverse phase HPLC and concentration was determined by UV absorbance at $237 \mathrm{nM}$ using a molar extinction coefficient of 30,500 (10). Standard mixtures or samples were treated with $100 \mu$ l of ethereal diazomethane [prepared from $\mathrm{N}, \mathrm{N}$, dimethyl-nitrosoguanidine in a millimolar diazomethane generator (Pierce, Rockford, IL)] containing $10 \%$ anhydrous methanol, for $3 \mathrm{~h}$ at room temperature in a Teflon capped vial. Solvent was evaporated under a nitrogen stream, the methyl esters were converted to trimethylsilyl ethers by reaction with $50 \mu$ l BSTFA (Pierce) overnight at room temperature. Samples were either analyzed immediately or stored at $-20^{\circ} \mathrm{C}$ in that reagent until use. Just before GC analysis, the BSTFA was evaporated under a nitrogen stream and the sample was dissolved in approximately $5 \mu \mathrm{l}$ of hexane.
Approximately $0.5 \mu \mathrm{l}$ of the solution was injected onto the capillary column using a $\mathbf{J} \& \mathrm{~W}$ (Folsom, CA) cool on-column injector coupled to a $1 \mathrm{~m}$ length of inactivated, but uncoated, capillary column. This was butted to a $6 \mathrm{~m} 0.25 \mathrm{~mm}$ id, crosslinked methyl silicone column (Hewlett-Packard Co., Palo Alto, CA) maintained at $185^{\circ} \mathrm{C}$ in a Hewlett-Packard 5880 gas chromatograph. The column was connected through a capillary-direct interface maintained at $280^{\circ} \mathrm{C}$ to a Hewlett-Packard 5970 mass selective detector operated in the scan mode. Head pressure on the column was maintained at 5 psi of helium resulting in a flow rate of $0.5 \mathrm{ml} / \mathrm{min}$. Source, lens, and detector settings were established by the manufacturer's autotune software. Ion chromatograms were integrated, post-run, using software provided by the manufacturer. Standard curves were generated by linear regression analysis for (area of HETE ion peak/area of RA ion peak) versus (mass of HETE/mass of RA). Using this procedure we have demonstrated a linear response from 0.3 to $3 \mathrm{nmol}$ of each HETE. Recovery of authentic standards added to plasma or serum averaged $89.5 \pm 2.6 \%$ (14).

Metabolism of radiolabeled arachidonic acid by washed platelets. Platelets isolated from $3.0 \mathrm{ml}$ platelet-rich plasma by centrifugation at $1500 \mathrm{rpm}$ for $10 \mathrm{~min}$ was brought to $10 \mu \mathrm{M}$ in EDTA. Platelets were washed with calcium-magnesium-free Hanks' balanced salt solution, $25 \mathrm{mM}$ Tris, pH 7.4, and resuspended in $3.0 \mathrm{ml}$ of that buffer. These washed platelets were incubated at $37^{\circ} \mathrm{C}$ and were brought to a final concentration of $1.0 \mathrm{mM}$ in calcium chloride just before use. Platelets were incubated with ${ }^{14} \mathrm{C}$-arachidonic acid $(60 \mu \mathrm{Ci} / \mu \mathrm{mol})$ at concentrations of 10,30 , and $100 \mu \mathrm{M}$ at $37^{\circ} \mathrm{C}$ in a final volume of 0.25 $\mathrm{ml}$. After $2.5 \mathrm{~min}$ the reactions were stopped by the addition of $1.0 \mathrm{ml}$ chloroform-methanol (1:2 vol/vol). Radiolabeled products and substrate were extracted essentially as described by Bligh and Dyer (15). 12-HETE was resolved from other products by thin-layer chromatography on silica gel $\mathrm{G}$ developed in hexanediethyl ether-acetic acid (50:50:1 vol/vol/vol) and radioactivity of spots comigrating with authentic standard was determined by liquid scintillation spectroscopy in Liquiscint. Mass of HETE produced was calculated from specific activity of the radiolabeled arachidonic acid, corrected for total recovery of radioactivity through the procedure.

\section{RESULTS}

Platelet-poor plasmas from both adults and cord blood contained little of any of the HETE (Table 1). The means for each of the HETE in both populations were $0.11 \mu \mathrm{M}$ or less. Although plasma from cord blood contained twice as much 5- or 15-HETE as adult plasma, these differences were not statistically significant. The average level of each of the HETE was significantly higher in serum than in plasma. 12-HETE was 10 -fold more concentrated in both adult and neonatal sera than plasmas (Table 2). The differences between adult and cord blood serum 12-HETE were not significant $(p>0.10)$, due to the large variance in each population, even though the adult average was $60 \%$ more than the neonatal average. There were virtually no differences between adult and neonatal sera with regard to either 5-HETE or 15HETE levels.

A striking difference was observed between the ability of adult and cord blood cells to produce 12-HETE when provided with arachidonic acid and A23187 (Fig. 1; Table 2). Serum from adults, generated in the presence of these compounds, contained an average of $21.3 \mu \mathrm{M}$ 12-HETE, whereas cord serum reached only $1.75 \mu \mathrm{M}(p<0.001)$. The lowest level of 12-HETE production in adult blood was greater than the highest level seen in cord blood (Fig. 2). When ratios between stimulated and basal serum levels were determined for individuals, the average adult serum formed in the presence of arachidonate and A23187 contained 33 times as much 12-HETE as unstimulated serum, whereas the average ratio for the neonate was only four.

These differences in 12-HETE levels apparently did not result 
Table 2. HETE content of plasma and serum from adults and neonates (mean $\pm S E)^{*}$

\begin{tabular}{|c|c|c|c|c|}
\hline \multirow[b]{2}{*}{ Source } & \multirow[b]{2}{*}{ Treatment } & \multicolumn{3}{|c|}{ HETE isomer $(\mathrm{nmol} / \mathrm{ml})$} \\
\hline & & 5-HETE & 12-HETE & 15-HETE \\
\hline \multirow[t]{3}{*}{ Adult } & Plasma & $0.051 \pm 0.021$ & $0.065 \pm 0.038$ & $0.018 \pm 0.012$ \\
\hline & Serum & $0.22 \pm 0.08$ & $0.82 \pm 0.22$ & $0.069 \pm 0.025$ \\
\hline & Stimulated serum & $3.64 \pm 0.48$ & $21.3 \pm 2.78$ & $1.01 \pm 0.36$ \\
\hline \multirow[t]{3}{*}{ Neonate } & Plasma & $0.11 \pm 0.055$ & $0.047 \pm 0.016$ & $0.041 \pm 0.012$ \\
\hline & Serum & $0.21 \pm 0.072$ & $0.55 \pm 0.11$ & $0.080 \pm 0.025$ \\
\hline & Stimulated serum & $2.61 \pm 0.496$ & $1.75 \pm 0.46$ & $1.11 \pm 0.37$ \\
\hline
\end{tabular}

* The indicate HETE isomer concentration was determined for each of the blood fractions for adult venous blood and neonatal cord blood.

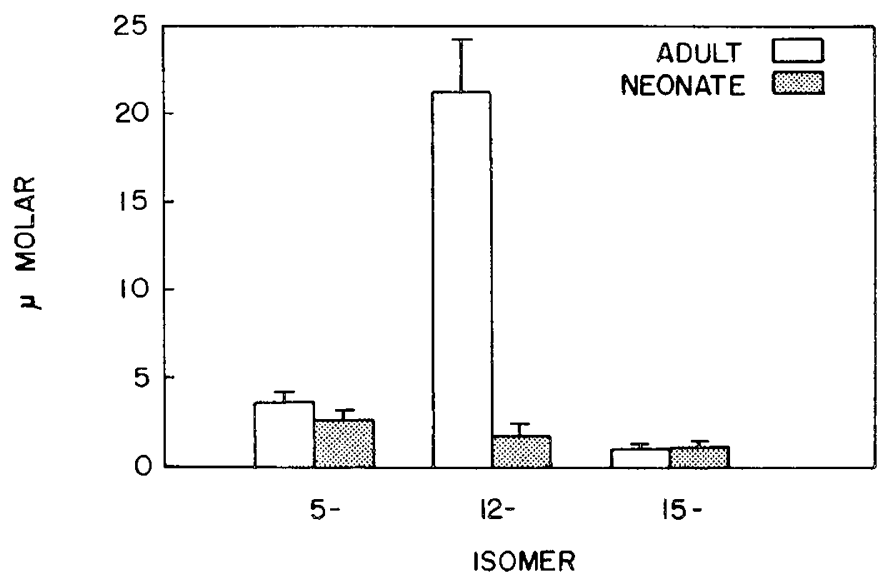

Fig. 1. HETE concentrations in stimulated sera from adults and neonates. Mean concentration (in $\mu \mathrm{M}$ ) of the indicated HETE positional isomer in stimulated serum obtained from adult blood (open bars) or cord blood (hatched bars) plus $1 \mathrm{SD}$ is depicted.

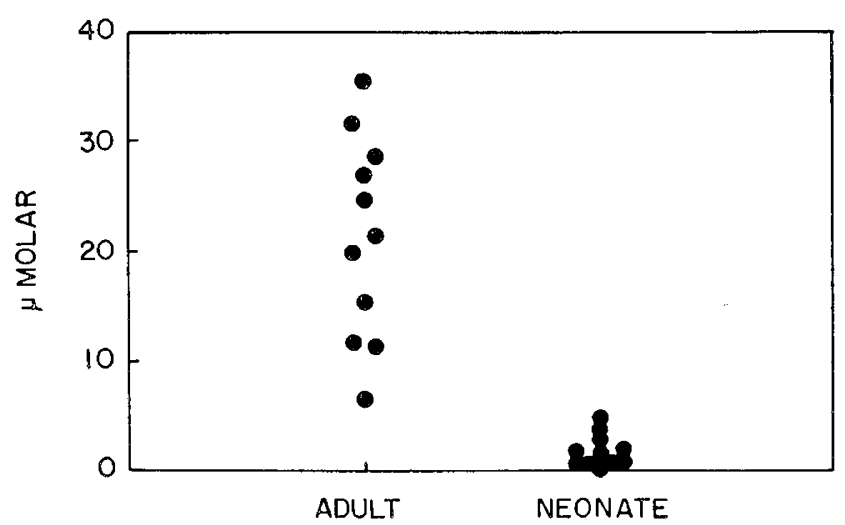

Fig. 2. 12-HETE concentrations in stimulated sera from individual adults and neonates.

from differences in the numbers of platelets in the blood from adults versus umbilical cords. Platelet counts in adult and neonate were nearly identical $\left(263 \pm 14\right.$ versus $294 \pm 19 \times 10^{3} / \mu 1$, respectively, $c f$. Table 1 ). Thus, neither the slight reduction in 12-HETE in cord blood plasma and serum nor the enormous reduction in stimulated cord serum (compared to adults) can be explained by a corresponding quantitative platelet abnormality. Cord blood contained an average of twice as many white blood cells as did adult blood $\left(12.7 \pm 1.4 \times 10^{3} / \mu 1\right.$ versus $6.3 \pm 0.6, c f$. Table 1). This difference might be responsible for the higher level of 5-HETE in plasma from neonates. However, despite the increased number of white cells, serum from cord blood, either spontaneously generated or formed during stimulation with arachidonate and ionophore, produced less or similar amounts of 5- and 15-HETE as did adult sera.

In exploring possible correlations between production of dif-

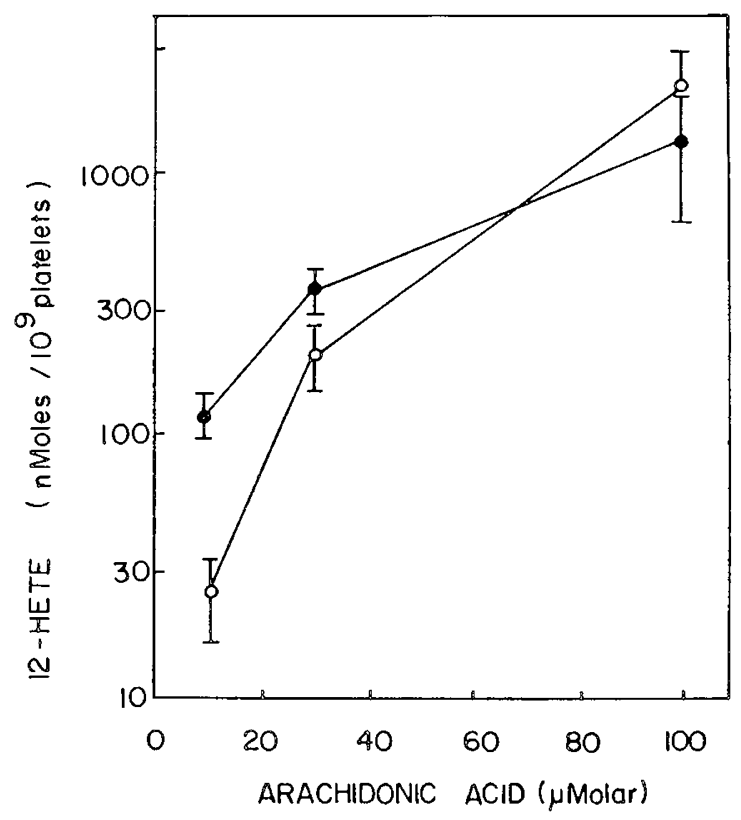

Fig. 3. Production of $12-\mathrm{HETE}$ by washed platelets from three adults (closed circles) and four neonates (open circles) (mean $\pm \mathrm{SD})$. Platelets were incubated for $150 \mathrm{~s}$ with the indicated concentration of $1-{ }^{14} \mathrm{C}$ arachidonic acid $(60 \mu \mathrm{Ci} / \mu \mathrm{mol})$. Note logarithmic ordinate.

ferent HETE, by the same or different fractions within individuals, only the following correlations were found. In adults, there was a positive correlation between production of 12 HETE in PPP and serum. That is, those individuals with the higher plasma levels of 12-HETE also tended to produce the higher levels of 12-HETE in their serum $(r=0.77)$. In addition, there was a correlation between 12- and 15-HETE levels in adult serum $(r=$ $0.74)$ and also in plasma $(r=0.79)$. In neonates, the only observed correlation was between the 5-HETE and 12-HETE content of plasma $(r=0.76)$.

We also investigated if different groups of neonates differed from the mean or from each other with regard to any of the HETE measured in the various blood preparations. Cord blood from female neonates produced nearly twice as much 12-HETE in stimulated serum as did blood from males $(2.1 \pm 1.7 \mu \mathrm{M}$ versus $1.1 \pm 1.1$ ) but the difference was not statistically significant $(p>0.2)$. No other differences were obvious between male and female neonates. When method of delivery was investigated, it was found that vaginally delivered infants had cord serum levels of 12-HETE twice those delivered by cesarean section $(1.08 \pm$ $0.52 \mu \mathrm{M}$ versus $0.45 \pm 0.29, p<0.05)$. No significant differences in the other HETE were observed in serum, and neither stimulated serum nor plasma HETE values differed among these two groups.

To determine if the altered production of 12-HETE in stimulated serum resulted from differences between adult and cord blood platelets themselves, rather than differences in other blood 
components, we examined the production of 12-HETE from arachidonic acid by washed platelets. The results depicted in Figure 3 demonstrate that cord blood platelets do differ from adult platelets in their ability to produce 12-HETE when provided with arachidonic acid. At $10 \mu \mathrm{M}$ arachidonic acid, well below the apparent $K_{\mathrm{m}}$ of lipoxygenase for that substrate (16), adult platelets produced nearly five times more 12-HETE than did neonatal platelets $\left(118 \pm 24 \mathrm{nmol} / 10^{9}\right.$ adult platelets versus $25 \pm 9$ for neonates. $p<0.01$ ). At $30 \mu \mathrm{M}$ arachidonate the disparity was not as great $\left(353 \pm 74 \mathrm{nmol} / 10^{9}\right.$ platelets for adults versus $199 \pm 55$ for neonates) and at $100 \mu \mathrm{M}$, neonatal platelets produced more 12-HETE than did adult platelets $(1300 \pm 68$ $\mathrm{nmol} / 10^{9}$ platelets for adults versus $2160 \pm 790$ for cord blood platelets) although these latter differences were not statistically significant $(p>0.10)$. It is worth noting that the levels of 12HETE produced by washed platelets from adults stimulated with $10 \mu \mathrm{M}$ arachidonic acid are similar to the amounts produced in stimulated serum. Assuming $0.25 \times 10^{9}$ platelets $/ \mathrm{ml}$ the washed platelets should produce 12-HETE concentrations of $29 \mu \mathrm{M}$ for adults (versus $21.3 \mu \mathrm{M}$ actually measured). However, the calculated value of $6 \mu \mathrm{M}$ for neonatal platelets was greater than the observed value of $1.75 \mu \mathrm{M}$ (Table 1 ).

\section{DISCUSSION}

Hydroxyeicosanoids have been found to be involved in a number of biologic responses. Perhaps the first of these to be characterized was the chemoattraction of neutrophils toward 12HETE (16). With the discovery of leukotrienes, important products of lipoxygenase-initiated metabolism in neutrophils, interest in the functions of HETE was reawakened. 5-HETE was found to be a stimulus of neutrophil secretory reactions (18). 15-HETE was found to modulate other reactions of arachidonic acid, inhibiting platelet 12-lipoxygeanse (19), but activating the 5lipoxygenase (20) in other cells. Our laboratory has discovered effects of HETE on cell motility and cell division. 15-HETE has been found to promote neovascularization, a process that is fundamental to many physiologic and pathologic states (6-8). 15-HETE $(0.1 \mathrm{mM})$ stimulates the migration of capillary endothelial cells in an in vitro Boyden chamber assay and also enhances cell proliferation. Further studies have also revealed an in vivo effect of 15-HETE on neovascularization in the rabbit corneal pocket assay $(16,17)$. Studies using lipoxygenase inhibitors have implicated HETE in vascular endothelial cell proliferation (10), and stimulus activation of macrophages (21) and neutrophils (22).

When the levels of HETE in plasma from normal adults or neonates was determined, the mean level of the HETE was not sufficient to evoke any of the known biologic responses. However, when blood was allowed to clot, the resulting levels of $12-$ HETE were sufficient to serve as chemoattractants for neutrophils $(1 \mu \mathrm{M})$, and levels of 15-HETE were similar to those demonstrated to cause endothelial cell migration and proliferation (9). Blood cells have the capacity to produce far more HETE than formed during clotting. When presented with excess substrate and the calcium ionophore A23187, production of 12 HETE by adult serum was stimulated to a mean of $22 \mu \mathrm{M}$ in 1 $h$. This level surpasses the concentration at which 12-HETE inhibits cyclooxygenase (11), or stimulates smooth muscle cell migration (9) and approaches the concentration shown to inhibit endothelial cell migration (6). 5-HETE also accumulated to a level at which stimulation of neutrophil secretion has been observed (18). Under these conditions, 15-HETE reached a concentration of over $1 \mu \mathrm{mol}$, similar to that required for activation of leukotriene synthesis (20).

Although plasma and serum samples from cord bloods contained similar levels of all the HETE measured (Table 2), in response to the additional stimuli arachidonic acid and A23187, neonatal blood produced only one-twelfth the amount of 12HETE as did adult blood. This discrepancy was unique to $12-$
HETE, inasmuch as adult and neonatal stimulated sera contained similar levels of 5- and 15-HETE. This discrepancy could result from any of at least three causes. First, lipoxygenase activity in neonatal platelets might be reduced or altered in kinetic properties. Second, neonatal blood might contain increased levels of proteins or other components that bind or otherwise remove arachidonic acid from access to the platelet. Third, neonatal blood might contain increased levels of components that bind, sequester, or metabolize the 12-HETE that was produced.

When we measured 12-HETE production by washed platelets, we observed that at $10 \mu \mathrm{M}$ arachidonic acid, neonatal platelets appeared to have only one-quarter the activity of their adult counterparts. When the concentration of substrate was raised to $100 \mu \mathrm{M}$, the difference disappeared. Whereas it is difficult to draw kinetic conclusions from whole cell experiments, these results are consistent with an increased $K_{\mathrm{m}}$ (decreased affinity) of neonatal platelet lipoxygenase for arachidonic acid, but a similar maximal level of enzyme activity when saturating substrate is available. If this explanation is the reason for the reduced 12-HETE level in neonatal stimulated sera, it also suggests that even at $2 \mu \mathrm{M}$ arachidonic acid and $25 \mu \mathrm{M}$ A23187, the concentration of substrate actually experienced by the platelet enzyme is quite low. It is possible that some component of neonatal serum further reduces the concentration of arachidonic acid available to the enzyme. However, it is unlikely that this arachidonic acid sink is plasma albumin or another plasma protein because we have previously shown that plasma from neonates has less "binding capacity" for arachidonic acid than that found in adult plasma (23).

The addition of arachidonic acid at much higher levels than is found in normal blood along with the calcium ionophore is certainly an artificial system. We have used it to determine the maximal level of HETE that might be produced by blood cells. Yet the levels of HETE produced in response to these extraordinary stimuli are not that different than might be produced in more physiologic settings. Collagen stimulation of platelet-rich plasma has been seen to produce 5 to $10 \mu \mathrm{M}$ 12-HETE (24).

Although 12-HETE has been implicated in some reports in the second phase or disaggregation phase of responses to stimuli (25), there is as yet no generally agreed on, clearly defined role for this compound in platelet function or hemostasis. The only reported pathologic entity in which 12-HETE production is reduced is the myeloproliferative syndrome (26). In this condition, an increased bleeding tendency has been correlated with the platelet 12-lipoxygenase deficiency. Whereas the normal neonate does not manifest a bleeding tendency and has a normal bleeding time (27), severe bleeding can occur in preterm infants $(28,29)$. Should 12-HETE play an heretofore undiscovered essential role in hemostasis, it is possible that pathologic bleeding in the neonate might result from further reduction in the already impaired platelet 12-HETE synthetic ability.

\section{REFERENCES}

1. Hamberg M, Samuelsson B 1974 Prostaglandin Endoperoxides: novel transformations of arachidonic acid in human platelets. Proc Natl Acad Sci USA $71: 3400-3444$

2. Borgeat P, Samuelsson B 1979 Arachidonic acid metabolism in polymorphonuclear leukocytes. Proc Natl Acad Sci USA 76:2148-2152

3. Bryant RW, Bailey JM, Schewe T, Rapoport SM 1982 Positional specificity of reticulocyte lipoxygenase. Conversion of arachidonic acid to 15-s-hydroperoxy-eicosatetraenoic acid. J Biol Chem 257:6050-6055

4. Setty BNY, Walenga RW, Stuart MJ 1985 Formation of 11- and 15-HETE by human umbilical arteries is catalyzed by cyclooxygenase. Biochim Biophys Acta 833:484-494

5. Hopkins NK, Oglesby TD, Bundy GL, Gorman RR 1984 Biosynthesis and metabolism of 15 -hydroperoxy-5,8,11,13-eicosatetraenoic acid by human umbilical vein endothelial cells. J Biol Chem 259:14048-14053

6. Graeber JE, Walenga RW, Conner TB, Stuart MJ, Glaser BM 1984 Hydroxyeicosatetraenoic acids (12- and 15-HETEs alter endothelial cell migration in vitro. Fed Proc 43:588

7. Graeber JE, Stuart MJ, Glaser BM 1987 15-Hydroxy-eicosatetraenoic acid promotes migration of human microvessel endothelial cells in vitro. Pediatr Res 21:214A

8. Graeber JE, Stuart MJ, Glaser BM 1986 15-Hydroxy-eicosatetraenoic acid is 
proangiogenic in vivo. Possible role in pathological neovascularization. $\mathrm{Pe}$ diatr Res 20:329A

9. Nakao J, W-C Chang HI, Koshihara Y, Murota S 1988 Aortic smooth muscle cell migration caused by platelet-derived growth factor is mediated by lipoxygenase products of arachidonic acid. Biochem Biophys Res Commun 112:866-871

10. Setty BNY, Dubowy RL, Stuart MJ 1987 Endothelial cell proliferation may be mediated via the production of endogenous lipoxygenase metabolites. Biochem Biophys Res Commun 144:345-351

11. Setty BNY, Stuart MJ 1987 15-Hydroxy-5,8,11,13-eicosatetraeneoic acid inhibits human vascular cyclooxygenase. J Clin Invest 77:202-211

12. Stuart MJ, Allen JB 1982 Arachidonic acid metabolism in the neonatal platelet. Pediatrics 69:714-718

13. Borgeat P, Hamberg M, Samuelsson B 1976 Transformation of arachidonic acid and homolinolenic acids by rabbit polymorphonuclear leukocytes. J Biol Chem 251:7816-7820

14. Walenga RW, Boone SB, Stuart MJ 1987 Analysis of blood HETE levels by selected ion monitoring with ricinoleic acid as the internal standard. Prostaglandins 34:733-748

15. Bligh EG, and Dyer WJ 1959 A rapid method for total lipid extraction and purification. Can J Biochem Physiol 37:911-917

16. Sun FF, McGuire JC, Metzler CM 1981 The effect of substrate availability on the metabolism of arachidonic acid in the human platelet. Prog Lipid Res 20:275-278

17. Goetzl EJ, Woods JM, Gorman RR 1977 Stimulation of human eosinophil and neutrophil polymorphonuclear leukocyte chemotaxis and random migration by 12 -L-hydroxy-5,8,11,14 eicosatetraenoic acid. J Clin Invest $59: 179-183$
18. Stenson WF, Parker C 1980 Monohydroxyeicosatetraenoic acids (HETEs) induce degranulation of human neutrophils. J Immunol 124:2100-2104

19. Vanderhoek JY, Bryant RW, Bailey JM 1980 15-Hydroxy-5,8,11,13-eicosatetraenoic acid. A potent and selective inhibitor of platelet lipoxygenase. $\mathrm{J}$ Biol Chem 255:5996-5998

20. Vanderhoek JY, Tare NS, Bailey JM, Goldstein AL, Pluznik DH 1982 New role for 15-hydroxyeicosatetraenoic acid activator of leukotriene biosynthesis in PT-18 mast/basophil cells. J Biol Chem 257:12191-12195

21. Schade UF 1986 Involvement of lipoxygenase in the activation of mouse macrophages by endotoxin. Biochem Biophys Res Commun 138:842-849

22. Dorsch W, Ring J, Riepel H 1984 Int Arch Allerg Appl Immunol 73:274-279

23. Sadowitz PD, Walenga RW, Clark D, Stuart MJ 1987 Decreased plasma arachidonic acid binding capacity in neonates. Biol Neonate 51:305-311

24. Hwang DH 1982 Characteristics of the formation of the platelet lipoxygenase product from endogenous arachidonic acid. Lipids 17:845-847

25. Dutihl CE, Haddeman E, ten Hoor F 1980 Role of lipoxygenase pathway in blood platelet aggregation. Adv Prostaglandin Thromboxane Leukotriene Res 6:101-106

26. Schafer AI 1982 Deficiency in platelet lipoxygenase in myeloproliferative disorders. N Engl J Med 306:381-386

27. Feusner JH 1980 Normal and abnormal bleeding times in neonates and young children using a fully standarized template technique. Am J Clin Patho 74:73-78

28. Rumack CM, Guggenheim MA, Rumack BH, Peterson RH, Johnson ML, Braithwaite WR 1981 Neonatal intracranial hemorrhage and maternal use of aspirin. Obstet Gynecol 58 (suppl)52S-56S

29. Hathaway WE, Bonnar J 1978 Perinatal Coagulation. Grune and Stratton, New York 